\title{
p16 promoter hypermethylation is associated with increased breast cancer risk
}

\author{
LI WANG, LIN TANG, RUILIAN XIE, WEIWEI NIE, LONGBANG CHEN and XIAOXIANG GUAN \\ Department of Medical Oncology, Jinling Hospital, Nanjing University School of Medicine, Nanjing 210002, P.R. China
}

Received March 25, 2012; Accepted July 18, 2012

DOI: $10.3892 / \mathrm{mmr} .2012 .1001$

\begin{abstract}
The current study aimed to investigate the effects of p16 hypermethylation on breast cancer quantitatively through a meta-analysis of available case-control studies (including malignant, benign and normal breast cancer tissues). The PubMed, Web of Science and EBSCO databases were searched from their inceptions to February 1, 2012. Crude odds ratios (ORs) with $95 \%$ confidence intervals (CIs) were extracted and pooled to assess the strength of the association between p16 hypermethylation and breast cancer risk. A total of eight studies, including 691 breast cancer cases and 525 control cases, were identified for meta-analysis. Statistically significant ORs of p16 hypermethylation were obtained from the breast cancer and control groups (OR, 6.58; 95\% CI, 1.15-37.75; $\mathrm{P}=0.03$ ). However, no significant associations between the methylation and ER and PR status in breast cancer were detected (OR, 1.24; 95\% CI, 0.64-2.41; P=0.52; OR, 1.49; 95\% CI, 0.81-2.75; $\mathrm{P}=0.20$, respectively). The meta-analysis indicated that $\mathrm{p} 16$ hypermethylation significantly increases breast cancer risk. However, no significant associations between the methylation and ER and PR status in breast cancer were detected.
\end{abstract}

\section{Introduction}

Breast cancer is currently one of the leading causes of mortality due to cancer in females worldwide. Approximately 230,480 new cases of invasive breast cancer and 39,520 deaths due to breast cancer were expected to occur among women in the USA in 2011 (1). Similar to other tumor types, breast carcinoma is thought to arise following the activation of oncogenes and inactivation of tumor suppressor genes. p16, one of the most commonly inactivated tumor suppressor genes in human cancer, is a cyclin-dependent kinase inhibitor that regulates

Correspondence to: Professor Xiaoxiang Guan, Department of Medical Oncology, Jinling Hospital, Nanjing University School of Medicine, 305 East Zhongshan Road, Nanjing, Jiangsu 210002, P.R. China

E-mail:xguan@nju.edu.cn

Key words: p16 hypermethylation, breast cancer, meta-analysis, estrogen receptor, progesterone receptor tumor progression through the G1 phase of the cell cycle (2). The downregulation of p16 expression due to promoter hypermethylation frequently occurs in breast cancer. A number of studies have shown that p16 hypermethylation is an early and likely critical step in breast cancer development $(3,4)$. Aberrant DNA hypermethylation has been increasingly recognized as a frequent molecular alteration in cancer $(5,6)$. This epigenetic modification occurs at the cytosines of $\mathrm{CpG}$ dinucleotide-rich regions ( $\mathrm{CpG}$ islands), which are mostly unmethylated in normal tissues. The hypermethylation of $\mathrm{CpG}$ islands in the gene promoter regions of numerous tumor suppressor and DNA repair genes is associated with chromatin condensation, replication delay, inhibition of the initiation of transcription and gene silencing (7). Similar to a number of other genes, p16 is commonly inactivated by the hypermethylation of its CpG-rich promoter region (8). The association between p16 promoter methylation and breast cancer susceptibility has been extensively studied; however, the results are inconsistent (8-16). Thus, a comprehensive meta-analysis on the most recent and relevant articles was performed to identify statistical evidence of the association between p16 gene hypermethylation and breast cancer risk. In addition, the correlation of p16 methylation with estrogen receptor (ER) and progesterone receptor (PR) status in breast cancer differed from those in previous studies. Therefore, a systematic review and meta-analysis was conducted to evaluate this correlation quantitatively.

\section{Materials and methods}

Meta-analysis. The meta-analysis was performed according to the Preferred Reporting Items for Systematic Reviews and Meta-Analysis (17) and the recommendations of the Cochrane Collaboration (18). The study was approved by the Research Ethics Committee of Jinling Hospital.

Identification and eligibility of relevant studies. To gather eligible articles, the terms 'p16,' 'methylation,' 'breast cancer,' 'breast tumor,' 'breast carcinoma,' 'estrogen receptor,' 'ER,' 'progesterone receptor' and 'PR' were searched for in PubMed, Web of Science and EBSCO with an English language restriction. The search results were updated until February 1, 2012. Only published full-text articles were included in the analysis. The following criteria were used in selecting eligible articles: (a) articles dealing with p16 methylation and breast cancer risk; (b) independent case-control, 
malignant and benign breast cancer tissue studies with reliable methods; and (c) sufficient data for estimating odds ratios (ORs) with 95\% confidence intervals (CIs). Articles concerning p16 methylation and the ER and PR status in breast cancer were also searched.

Data extraction. Wang and Nie independently conducted reviews by extracting data via a standardized approach. The publication information (year of publication and name of first author), study characteristics (sample size and distribution of ER and PR) and p16 hypermethylation rates were collected using standard data extraction forms. The discrepancies were resolved through discussions. Subgroup analyses were stratified using the ER and PR status in breast cancer cases.

Statistical analysis. The ORs and 95\% CIs were calculated to estimate the strength of the association between p16 hypermethylation and breast cancer risk. A Chi-square-based Q-test was performed to assess between-study heterogeneity. If $\mathrm{P}>0.10$, the studies were considered to lack heterogeneity and the pooled ORs were calculated using the fixed-effects model according to the Mantel-Haenszel method. Otherwise, the random-effect model was used according to the DerSimonian-Laird method. A sensitivity analysis was performed to assess the stability of the meta-analysis results by repeating the meta-analysis and omitting one study at each iteration (19). Begg's funnel plot and Egger's linear regression test were used to estimate potential publication bias. An asymmetric funnel plot indicated possible publication. In Egger's test, $\mathrm{P}<0.05$ indicated the existence of statistically significant publication bias. The shapes of the funnel plots did not reveal any evidence of asymmetry (Fig. 1) and the Egger's test results did not suggest any evidence of publication bias $(\mathrm{P}>1.00)$. All statistical analyses in the current study were performed using Review Manager 5 and Stata 11.0 software.

\section{Results}

Study characteristics. A total of eight articles met the inclusion criteria and were used in the current meta-analysis. The studies involved 691 breast cancer cases and 525 controls. When the same investigators reported results obtained from the same cohort of patients in several publications, only the largest series was included in the analysis. A cohort of patients was excluded due to duplicate reports (20). Only four articles were included in the analysis for the quantitative evaluation of the correlation between p16 hypermethylation and the ER and PR status in breast cancer $(2,11,13,21)$. The main characteristics of these studies are listed in Tables I-III.

\section{Main results}

p16 hypermethylation in breast cancer and control groups. Data for the p16 hypermethylation comparison among the breast cancer and control groups were taken from eight studies. The breast cancer and control groups included 227 (32.9\%) and 25 (4.8\%) p16 hypermethylated cases, respectively. The pooled analysis shows that the ORs of the breast cancer group during p16 hypermethylation significantly increased compared with those of the controls (OR, 6.58; 95\% CI, 1.15-37.75; $\mathrm{P}=0.03)$. However, the studies were heterogeneous $(\mathrm{P}<0.0001$,

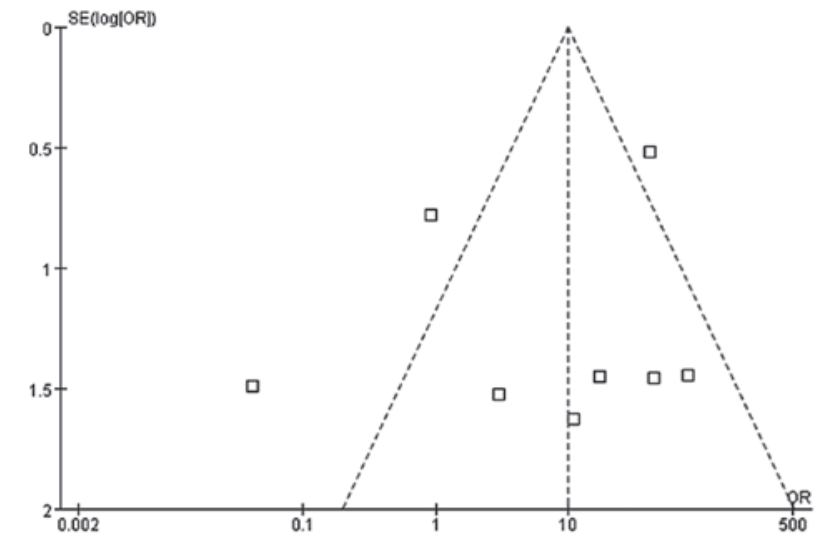

Figure 1. The funnel plot with $95 \%$ CIs of studies on methylation of the p16 gene and breast cancer showing no asymmetry. CI, confidence interval.

$\mathrm{I}^{2}=80 \%$, Fig. 2 ) and this heterogeneity was incorporated into the random-effects model. The funnel plots did not show any evidence of publication bias.

Correlation between p16 methylation and the ER and PR status in breast cancer. A total of four studies calculated the ORs of p16 hypermethylation in ER-positive and -negative breast cancer (Fig. 3). Of the 308 hypermethylated breast cancer cases, 230 were ER-positive (74.7\%), whereas among the 877 unmethylated breast cancer cases, 641 were ER-positive (73.1\%). The pooled analysis revealed no significantly increased ORs in the correlation between the ER and methylation status in breast cancer (OR, 1.24; 95\% CI, 0.642.41; $\mathrm{P}=0.52)$. However, 210 out of the 302 hypermethylated breast cancer cases were PR-positive (69.5\%), whereas 555 out of the 866 unmethylated breast cancer cases were PR-positive (64.1\%). The pooled analysis revealed no significantly increased ORs in the correlation between the PR and methylation status in breast cancer (OR, 1.49; 95\% CI, 0.81-2.75; $\mathrm{P}=0.20)$. However, the studies were heterogenous $(\mathrm{P}<0.05$, Figs. 3 and 4) and this heterogeneity was incorporated into the random-effects model. Funnel plots did not show any evidence of publication bias.

\section{Discussion}

Breast cancer is the most common cancer among women and is the second leading cause of mortality worldwide (22). Its occurrence is associated with genetic and environmental risk factors. $\mathrm{CpG}$ islands are located in the promoter or first exon region of genes and are normally unmethylated. However, in cancer cells, the hypermethylation of these regions is associated with transcriptional silencing or a decrease in expression. The promoter hypermethylation of tumor suppressor genes, including p16, hMLH1 and VHL, has been established as a common mechanism for tumor suppressor inactivation in human cancer and has become a promising new molecular target for its detection (8). Aberrant DNA methylation has been increasingly recognized as a frequent molecular alteration in breast cancer (11-14). Genes involved in cell-cycle regulation (p16), cell adhesion (CDH1), DNA repair (BRCA1) and cell signaling pathways (ER and RAR $\beta$ ) have been reported to 
Table I. Data of p16 methylation included in this study.

\begin{tabular}{|c|c|c|c|c|c|}
\hline \multirow[b]{2}{*}{ First author (ref.) } & \multirow[b]{2}{*}{ Year } & \multicolumn{2}{|c|}{ Case (n) } & \multicolumn{2}{|c|}{ Control (n) } \\
\hline & & Methylated & Unmethylated & Methylated & Unmethylated \\
\hline Di Vinci A (8) & 2005 & 9 & 5 & 10 & 5 \\
\hline Cavusoglu CA (10) & 2010 & 15 & 47 & 0 & 4 \\
\hline Jing F (11) & 2008 & 30 & 72 & 0 & 20 \\
\hline Jing F (12) & 2010 & 15 & 35 & 0 & 50 \\
\hline Raish M (13) & 2009 & 112 & 239 & 4 & 347 \\
\hline Vallian S (14) & 2009 & 25 & 45 & 0 & 70 \\
\hline Van Zee KJ (15) & 1998 & 16 & 17 & 11 & 0 \\
\hline Woodcock DM (16) & 1999 & 5 & 4 & 0 & 4 \\
\hline
\end{tabular}

Table II. Data of p16 methylation by various ER statuses included in this study.

\begin{tabular}{|c|c|c|c|c|c|}
\hline \multirow[b]{2}{*}{ First author (ref.) } & \multirow[b]{2}{*}{ Year } & \multicolumn{2}{|c|}{ Methylated (n) } & \multicolumn{2}{|c|}{ Unmethylated (n) } \\
\hline & & $\mathrm{ER}(+)$ & $\mathrm{ER}(-)$ & $\mathrm{ER}(+)$ & $\mathrm{ER}(-)$ \\
\hline Jing F (11) & 2008 & 20 & 10 & 33 & 39 \\
\hline $\mathrm{Li} \mathrm{S}(21)$ & 2006 & 18 & 8 & 116 & 46 \\
\hline Таo MH (2) & 2009 & 92 & 48 & 299 & 105 \\
\hline Raish M (13) & 2009 & 100 & 12 & 193 & 46 \\
\hline
\end{tabular}

ER, estrogen receptor.

Table III. The data of methylation of p16 in different PR statuses included in this study

\begin{tabular}{|c|c|c|c|c|c|}
\hline \multirow[b]{2}{*}{ First author (ref.) } & \multirow[b]{2}{*}{ Year } & \multicolumn{2}{|c|}{ Methylated (n) } & \multicolumn{2}{|c|}{ Unmethylated (n) } \\
\hline & & $\operatorname{PR}(+)$ & PR(-) & $\operatorname{PR}(+)$ & $\operatorname{PR}(-)$ \\
\hline Jing F (11) & 2008 & 21 & 9 & 28 & 44 \\
\hline Li S (21) & 2006 & 18 & 8 & 102 & 60 \\
\hline Tao MH (2) & 2009 & 78 & 56 & 247 & 146 \\
\hline Raish M (13) & 2009 & 93 & 19 & 178 & 61 \\
\hline
\end{tabular}

$\mathrm{PR}$, progesterone receptor.

undergo hypermethylation $(23,24)$. The product of INK4A locus, p16, encodes a cyclin-dependent kinase (CDK) inhibitor that functions as a negative regulator of cyclin/CDK complexes. It binds preferentially to CDK4/6 and prevents the association of CDK4/6 with D-type cyclins, thus inhibiting pRB phosphorylation and progression through the cell cycle $(25,26)$. p16 also plays a significant role in the maintenance of normal cellular properties and prevention of centrosome dysfunction and genomic instability (27). p16 inactivation occurs at the early stage of carcinogenesis $(25,26)$ and the loss of $\mathrm{p} 16 / \mathrm{Rb}$ activity occurs through various mechanisms, including the deletion, mutation and hypermethylation of p16 (27). p16 inactivation appears to be crucial in the development of several tumors (13). However, its relevance to mammary carcinogen- esis remains unclear. Moreover, the homozygous deletions of p16 are observed in half of the breast cancer cell lines and neither homozygous deletions nor point mutations are frequently observed in primary breast cancers, suggesting that these alterations were acquired in the cultures (13). Aberrant hypermethylation has been suggested to be a useful biomarker, with implications for breast cancer etiology, diagnosis and management. Gene-specific promoter alterations are common epigenetic aberrations in human breast cancer. However, the epigenetic changes in 16 gene hypermethylation specific to breast cancer etiology remain elusive. The current pooled analysis comprehensively assessed the correlation between p16 gene hypermethylation and the incidence of breast cancer based on eight studies, which included 691 breast cancer cases 


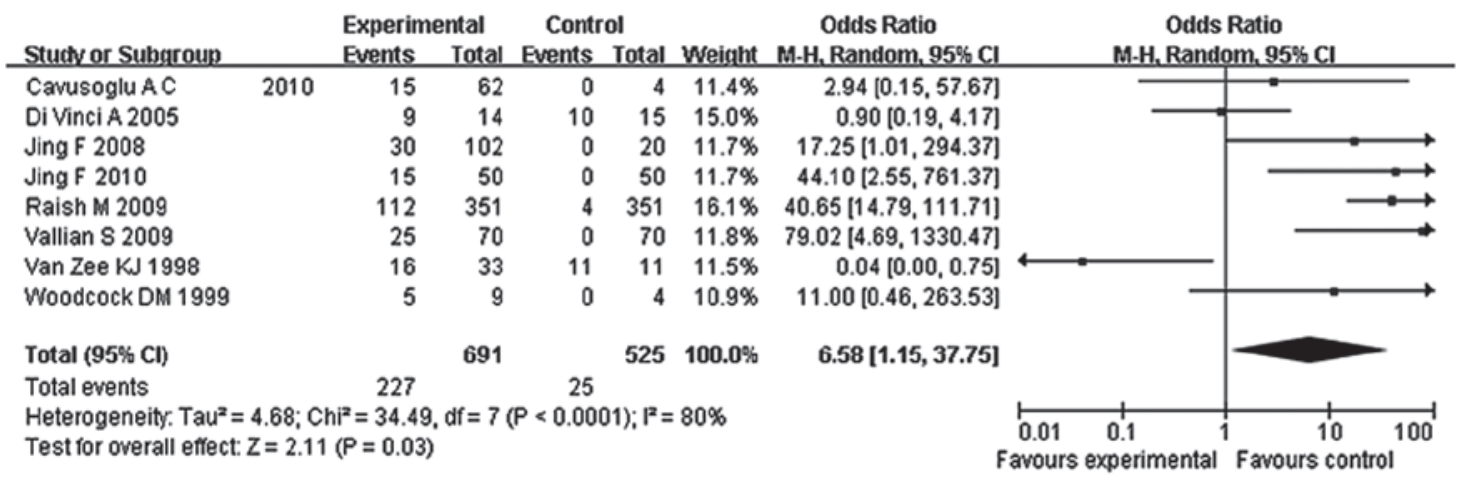

Figure 2. Pooled analysis of p16 hypermethylation in breast cancer and control group. CI, confidence interval.

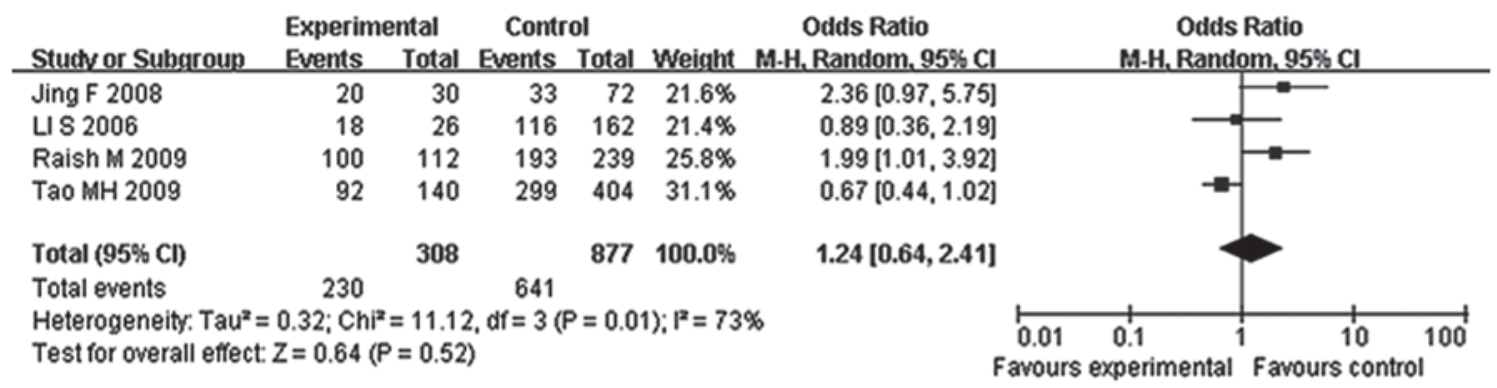

Figure 3. Pooled analysis of p16 hypermethylation in ER-positive and -negative breast cancer. ER, estrogen receptor; CI, confidence interval.

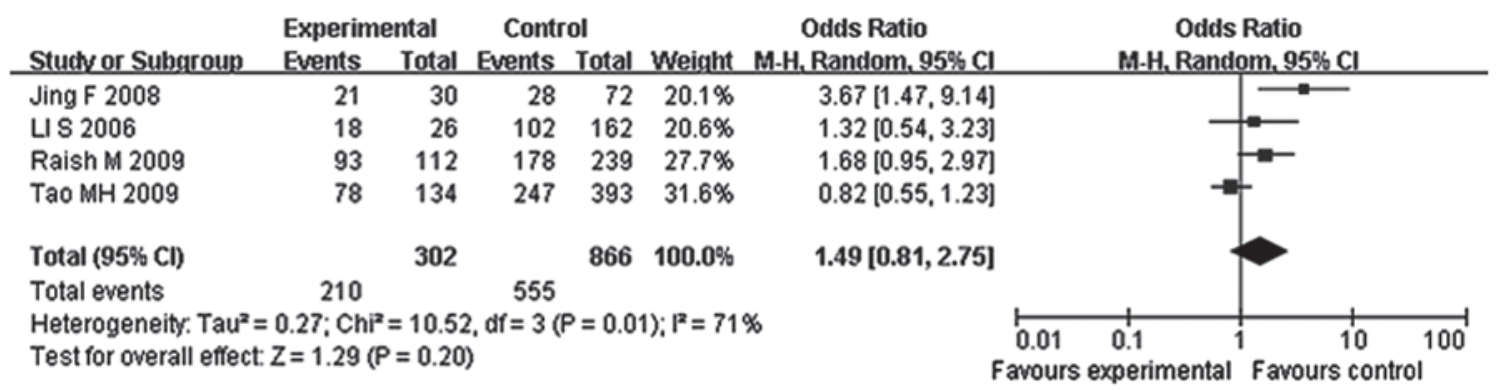

Figure 4. Pooled analysis of p16 hypermethylation in PR-positive and -negative breast cancer. PR, progesterone receptor; CI, confidence interval.

and 525 controls. Using the pooled crude ORs from studies, we identified that p16 gene hypermethylation is associated to 6.58-fold increased risks of breast cancer compared with the control group. Moreover, no significant association was identified among ER, PR and methylation status in breast cancer.

The current study has several potential limitations. First, the possibility of information and selection biases and unidentified confounders cannot be completely excluded as all studies were observational. Second, the searching strategy was restricted to English articles and thus, non-English articles with potentially high-quality data were not included due to anticipated difficulties in obtaining accurate medical translation. Third, most of the studies included in this meta-analysis were conducted in various ethnicities. Fourth, the results of the current study were not further validated.

In conclusion, despite the discrepancy in the p16 hypermethylation in the breast cancer and control groups, we found that p16 hypermethylation is associated with increased risk of breast cancer. Jing et al (11) demonstrated that p16 methylation is associated with the PR status. Tao et al (2) did not identify any significant differences in p16 methylation patterns relevant to the ER and PR status of breast cancer for postmenopausal women. The authors identified that p16 hypermethylation tended to be more frequent among ER-negative cases than ER-positive cases (OR, 1.51; 95\% CI, 1.01-2.32). Various studies $(2,11,13,21)$ did not identify any significant differences in tumor-related gene methylation patterns relevant to the ER and PR status of breast tumors. This meta-analysis indicates that the ER, PR and methylation status of the p16 gene in breast cancer are not significantly associated with one another. p16 hypermethylation, which mediates the inactivation of the p16 gene, plays a significant role in breast carcinogenesis. Therefore, it may have useful clinical applications in the detection of breast cancer at the early stages. In addition, current cancer 
therapies use DNA methylation inhibitors, including 5-aza2'-deoxycytidine (5-Aza-CdR), to reactivate the repressed p16 gene. The reactivation of the p16 gene in cells with methylated genes restores normal cell growth control (28-30). According to the current study, the p16 promoter methylation status could be used for breast cancer diagnostic and therapeutic purposes.

\section{Acknowledgements}

This project was supported by grants from National Natural Science Foundation of China (81141094, 30870962, to X. Guan), Natural Science Foundation of Jiangsu Province (BK2011656, to X. Guan).

\section{References}

1. DeSantis C, Siegel R, Bandi P and Jemal A: Breast cancer statistics, 2011. CA Cancer J Clin 61:409-418, 2011.

2. Tao MH, Shields PG, Nie J, et al: DNA hypermethylation and clinicopathological features in breast cancer: the Western New York Exposures and Breast Cancer (WEB) Study. Breast Cancer Res Treat 114: 559-568, 2009.

3. Holst CR, Nuovo GJ, Esteller M, et al: Methylation of p16(INK4a) promoters occurs in vivo in histologically normal human mammary epithelia. Cancer Res 63: 1596-1601, 2003.

4. Tlsty TD, Crawford YG, Holst CR, et al: Genetic and epigenetic changes in mammary epithelial cells may mimic early events in carcinogenesis. J Mammary Gland Biol Neoplasia 9: 263-274, 2004.

5. Wajed SA, Laird PW and DeMeester TR: DNA methylation: An alternative pathway to cancer. Annals Surg 234: 10-20, 2001.

6. Baylin SB and Herman JG: DNA hypermethylation in tumorigenesis - epigenetics joins genetics. Trends Genet 16: 168-174, 2000.

7. Esteller M, Corn PG, Baylin SB and Herman JG: A gene hypermethylation profile of human cancer. Cancer Res 61: 3225-3229, 2001.

8. Di Vinci A, Perdelli L, Banelli B, et al: p16(INK4a) promoter methylation and protein expression in breast fibroadenoma and carcinoma. Int J Cancer 114: 414-421, 2005.

9. Lehmann U, Langer F, Feist H, Glockner S, Hasemeier B and Kreipe H: Quantitative assessment of promoter hypermethylation during breast cancer development. Am J Pathol 160: 605-612, 2002.

10. Cavusoglu AC, Sevinc AI, Saydam S, et al: Promoter methylation and expression changes of $\mathrm{CDH} 1$ and $\mathrm{P} 16$ genes in invasive breast cancer and adjacent normal breast tissue. Neoplasma 57: 465-472, 2010

11. Jing F, Jun L, Yong Z, et al: Multigene methylation in serum of sporadic Chinese female breast cancer patients as a prognostic biomarker. Oncology 75: 60-66, 2008.

12. Jing $\mathrm{F}$, Yuping $\mathrm{W}$, Yong $\mathrm{C}$, et al: $\mathrm{CpG}$ island methylator phenotype of multigene in serum of sporadic breast carcinoma. Tumour Biol 31: 321-331, 2010.
13. Raish M, Dhillon VS, Ahmad A, et al: Promoter hypermethylation in tumor suppressing genes p16 and FHIT and their relationship with estrogen receptor and progesterone receptor status in breast cancer patients from Northern India. Transl Oncol 2: 264-270, 2009.

14. Vallian S, Sedaghat M, Nassiri I and Frazmand A: Methylation status of p16 (INK4A) tumor suppressor gene in Iranian patients with sporadic breast cancer. J Cancer Res Clin Oncol 135: 991-996, 2009.

15. Van Zee KJ, Calvano JE and Bisogna M: Hypomethylation and increased gene expression of p16(INK4a) in primary and metastatic breast carcinoma as compared to normal breast tissue. Oncogene 16: 2723-2727, 1998.

16. Woodcock DM, Linsenmeyer ME, Doherty JP and Warren WD: DNA methylation in the promoter region of the p16 (CDKN2/ MTS-1/INK4A) gene in human breast tumours. Br J Cancer 79: 251-256, 1999.

17. Moher D, Liberati A, Tetzlaff J, Altman DG and Grp P: Preferred reporting items for systematic reviews and meta-analyses: The PRISMA statement (Reprinted from Annals of Internal Medicine). Physical Therapy 89: 873-880, 2009.

18. Bero L and Rennie D: The Cochrane Collaboration. Preparing, maintaining, and disseminating systematic reviews of the effects of health-care. JAMA 274: 1935-1938, 1995.

19. Higgins JPT, Thompson SG, Deeks JJ and Altman DG: Measuring inconsistency in meta-analyses. BMJ 327: 557-560, 2003.

20. Jing F,Zhang J, Tao J, et al: Hypermethylation of tumor suppressor genes BRCA1, p16 and 14-3-3 sigma in serum of sporadic breast cancer patients. Onkologie 30: 14-19, 2007.

21. Li SY, Rong MN and Iacopetta B: DNA hypermethylation in breast cancer and its association with clinicopathological features. Cancer Lett 237: 272-280, 2006.

22. Parkin DM, Bray F, Ferlay J and Pisani P: Global cancer statistics, 2002. CA Cancer J Clin 55: 74-108, 2005.

23. Yang X, Yan L and Davidson NE: DNA methylation in breast cancer. Endocr Relat Cancer 8: 115-127, 2001.

24. Szyf M, Pakneshan P and Rabbani SA: DNA methylation and breast cancer. Biochem Pharmacol 68: 1187-1197, 2004.

25. Rocco JW and Sidransky D: p16(MTS-1/CDKN2/INK4a) in cancer progression. Exp Cell Res 264: 42-55, 2001.

26. Sherr CJ and McCormick F: The RB and p53 pathways in cancer. Cancer Cell 2: 103-112, 2002.

27. McDermott KM, Zhang JM, Holst CR, Kozakiewicz BK, Singla V and Tlsty TD: p16(INK4a) prevents centrosome dysfunction and genomic instability in primary cells. PLoS Biol 4: 350-365, 2006.

28. Bender CM, Pao MM and Jones PA: Inhibition of DNA methylation by 5-aza-2'-deoxycytidine suppresses the growth of human tumor cell lines. Cancer Res 58: 95-101, 1998.

29. Zhang B, Huang T, Liu K, Chen J and Wang G: Effects of 5-Aza-CdR on cell proliferation of breast cancer cell line MDA-MB-435S and expression of maspin gene. J Huazhong Univ of Sci Technology Med Sci 27: 543-546, 2007.

30. David GL, Yegnasubramanian S, Kumar A, et al: MDR1 promoter hypermethylation in MCF7 human breast cancer cells - Changes in chromatin structure induced by treatment with 5 -aza-cytidine. Cancer Biol Ther 3: 540-548, 2004. 